\title{
Regional contrast agent quantification in a mouse model of myocardial infarction using 3D cardiac $\mathrm{T}_{1}$ mapping
}

Bram F Coolen, Tessa Geelen, Leonie EM Paulis, Klaas Nicolay and Gustav J Strijkers

\begin{abstract}
Background: Quantitative relaxation time measurements by cardiovascular magnetic resonance (CMR) are of paramount importance in contrast-enhanced studies of experimental myocardial infarction. First, compared to qualitative measurements based on signal intensity changes, they are less sensitive to specific parameter choices, thereby allowing for better comparison between different studies or during longitudinal studies. Secondly, $\mathrm{T}_{1}$ measurements may allow for quantification of local contrast agent concentrations. In this study, a recently developed $3 \mathrm{D} \mathrm{T}_{1}$ mapping technique was applied in a mouse model of myocardial infarction to measure differences in myocardial $T_{1}$ before and after injection of a liposomal contrast agent. This was then used to assess the concentration of accumulated contrast agent.
\end{abstract}

Materials and methods: Myocardial ischemia/reperfusion injury was induced in 8 mice by transient ligation of the LAD coronary artery. Baseline quantitative $T_{1}$ maps were made at day 1 after surgery, followed by injection of a Gd-based liposomal contrast agent. Five mice served as control group, which followed the same protocol without initial surgery. Twenty-four hours post-injection, a second $T_{1}$ measurement was performed. Local $\Delta R_{1}$ values were compared with regional wall thickening determined by functional cine CMR and correlated to ex vivo Gd concentrations determined by ICP-MS.

Results: Compared to control values, pre-contrast $T_{1}$ of infarcted myocardium was slightly elevated, whereas $T_{1}$ of remote myocardium did not significantly differ. Twenty-four hours post-contrast injection, high $\Delta \mathrm{R}_{1}$ values were found in regions with low wall thickening values. However, compared to remote tissue (wall thickening $>45 \%$ ), $\Delta R_{1}$ was only significantly higher in severe infarcted tissue (wall thickening $\left.<15 \%\right)$. A substantial correlation $(r=$ 0.81) was found between CMR-based $\Delta R_{1}$ values and Gd concentrations from ex vivo ICP-MS measurements. Furthermore, regression analysis revealed that the effective relaxivity of the liposomal contrast agent was only about half the value determined in vitro.

Conclusions: $3 \mathrm{D}$ cardiac $T_{1}$ mapping by $C M R$ can be used to monitor the accumulation of contrast agents in contrast-enhanced studies of murine myocardial infarction. The contrast agent relaxivity was decreased under in vivo conditions compared to in vitro measurements, which needs consideration when quantifying local contrast agent concentrations.

\section{Background}

Mouse models of myocardial infarction are widely used to investigate the cascade of events occurring after myocardial ischemia/reperfusion injury. Cardiovascular magnetic resonance (CMR) has proven to be the modality of

\footnotetext{
* Correspondence: g.j.strijkers@tue.nl

Biomedical NMR, Department of Biomedical Engineering, Eindhoven University of Technology, PO BOX 513, 5600MB, Eindhoven, the Netherlands
}

choice for such studies, as it can produce high-resolution information on both global and local indices of cardiac pathology [1-3]. Also, MRI contrast agents are increasingly applied as they allow monitoring of various kinds of disease-related processes on cellular and even molecular level, such as cell death, inflammation and fibrosis [4-6].

For commonly used $\mathrm{T}_{1}$-weighted imaging, the accumulation of paramagnetic contrast agent is associated

\section{Biomed Central}


with local changes in signal intensity. However, signal intensity is an indirect and qualitative measure of concentration, because it is influenced by several other factors, such as coil sensitivities, gain calibration, sequence timing parameters and fluctuations in ECG- or respiratory triggering intervals. Consequently, there is a growing interest for the application of $T_{1}$ mapping protocols in contrast-enhanced studies of myocardial infarction using $\mathrm{T}_{1}$ contrast agents. Bohl et al. [7] used a LookLocker $\mathrm{T}_{1}$ mapping method to rapidly measure changes in $T_{1}$ in both remote and infarct areas after injection of Gd-DTPA. Quantitative knowledge of $\mathrm{T}_{1}$ was translated to the inversion time that resulted in optimal contrast in late Gd enhancement (LGE) imaging. Similar implementations of this method have also been used to dynamically measure $\mathrm{T}_{1}$ after infusion of $\mathrm{Mn}^{2+}$, an ion that acts as a $\mathrm{Ca}^{2+}$ analogue, to report on $\mathrm{Ca}^{2+}$ handling $[8,9]$.

In this study, we investigated whether quantitative $T_{1}$ measurements can also serve for the assessment of local contrast agent concentrations. This is particularly valuable for molecular imaging, where one aims to relate local amounts of contrast agent to the presence of certain disease markers. We recently presented a novel imaging protocol for measuring mouse myocardial $\mathrm{T}_{1}$ with whole-heart coverage, based on retrospective triggering and variable flip angle analysis [10]. Good reproducibility of measuring regional $\mathrm{T}_{1}$ was found between measurements at successive days; however, limited data was available concerning changes in $T_{1}$ in infarcted myocardium upon injection of a Gd-based contrast agent. Here, we hypothesize that this method provides an accurate readout of regional contrast agent induced changes of myocardial $\mathrm{T}_{1}$ in a mouse model of ischemia/reperfusion injury and allows for determination of local contrast agent concentrations. To test this hypothesis, we measured the accumulation of a liposomal contrast agent after myocardial ischemia/perfusion injury and quantified local changes in $\mathrm{T}_{1}$ using three-dimensional $\mathrm{T}_{1}$ mapping. Then, $\Delta \mathrm{R}_{1}$ values derived from pre- and post-contrast in vivo $\mathrm{T}_{1}$ maps were correlated to contrast agent concentrations obtained from ex vivo inductively coupled plasma mass spectrometry (ICP-MS).

The use of a liposomal contrast agent allowed for $T_{1}$ measurements several hours after contrast agent injection at a time point at which the contrast agent was almost cleared from the blood circulation and did not contaminate the measurement of myocardial $\mathrm{T}_{1}$. In this application, myocardial contrast agent concentrations were stable for an extended period of time, which increased the available imaging time and allowed for full 3D coverage of the heart. Moreover, the use of liposomes in this experimental mouse model of myocardial infarction provided the opportunity to perform relevant validation experiments concerning in vivo quantification of the actual contrast agent concentration and relaxivity properties in the heart.

\section{Materials and methods \\ Contrast agent preparation and characterization Preparation}

Paramagnetic liposomes were prepared as described earlier [11]. In short, liposomes were prepared by lipid film hydration of a lipid mixture containing $100 \mu \mathrm{mol}$ of total lipid. This mixture consisted of gadolinium-diethylenetriaminepentaaceticacid-bis(stearylamide) (GdDTPA-BSA, Gateway Chemical Technology, St. Louis, MO, USA), 1,2-distearoyl-sn-glycero-3-phosphocholine (DSPC, Lipoid GmbH, Ludwigshafen, Germany), cholesterol (Avanti Polar Lipids, Alabaster, AL, USA), 1,2-distearoyl-sn-glycero-3-phospoethanolamine- $N$-[methoxy (poly(ethylene glycol))-2000] (PEG2000-DSPE, Lipoid $\mathrm{GmbH}$ ) and 1,2-distearoyl-sn-glycero-3-phospoethanolamine- $N$-[maleimide(poly (ethylene glycol))-2000] (MalPEG2000-DSPE, Avanti Polar Lipids) at a molar ratio of $0.75 / 1.1 / 1 / 0.075 / 0.075$. The resulting vesicles were sized by extrusion through $200 \mathrm{~nm}$ filters (four times), followed by extrusion through two $100 \mathrm{~nm}$ filters (ten times). Liposomes were concentrated by centrifugation at $55000 \mathrm{rpm}$ and $4^{\circ} \mathrm{C}$ for 1 hour. Finally, liposomes were resuspended in HEPES-buffered saline $(20 \mathrm{mM}$ HEPES and $135 \mathrm{mM} \mathrm{NaCl}, \mathrm{pH}$ 7.4) at a concentration of approximately $70 \mathrm{mM}$ of total lipid.

\section{Characterization}

The final total lipid concentration was determined with a phosphate analysis according to Rouser [12]. Dynamic light scattering (DLS) was performed at $23^{\circ} \mathrm{C}$ with a Malvern ZetaSizer Nano S and the resulting hydrodynamic diameter of the liposomes was approximately 115 $\mathrm{nm}$. Longitudinal relaxivity $\mathrm{r}_{1}$ at $9.4 \mathrm{~T}$ was measured in HEPES-buffered saline at $37^{\circ} \mathrm{C}$. For this purpose, $\mathrm{T}_{1}$ measurements were performed on samples with various concentrations of Gd-DTPA-BSA (0.01-1.25 mM) using a snapshot-FLASH $\mathrm{T}_{1}$ mapping method [13]. The following parameters were used: scan repetition time $=15$ $\mathrm{s}, \mathrm{TR} / \mathrm{TE}=4.0 / 2.0 \mathrm{~ms}, \alpha=15^{\circ}$, Nsegments $=32$, slice thickness $=1 \mathrm{~mm}$. A total of 60 inversion times were sampled ranging from 72 to $4792 \mathrm{~ms}$.

\section{In vivo measurements}

Mice were obtained from a commercial breeder (Charles River, Paris, France) and housed under controlled conditions. A total of 13 Swiss mice (male, $33 \pm 2 \mathrm{~g}$ ) were used in this study. In the infarct group $(\mathrm{N}=8)$, mice underwent ischemia/reperfusion surgery by a 30 -minute ligation of the left anterior descending (LAD) coronary artery. One day after surgery, CMR was performed, consisting of $3 \mathrm{D} \mathrm{T}_{1}$ mapping measurements to assess 
baseline $T_{1}$, as well as 3D CINE measurements for determination of wall- thickening. This was followed immediately by injection of a liposomal contrast agent through the tail vein $(0.05 \mathrm{mmol} \mathrm{Gd} / \mathrm{kg})$. Five control mice served as control group, in which the same CMR measurements and contrast agent administration were performed without previous surgery. Twenty-four hours post-injection, a second $T_{1}$ measurement was performed using the same protocols. The mice were then sacrificed and the heart was excised to measure the Gd content ex vivo. The study protocols were approved by the local animal ethical committee.

CMR was performed with a 9.4 $\mathrm{T}$ Bruker experimental scanner using a 72-mm-diameter volume transmit coil in combination with a mouse cardiac phased-array surface coil (Bruker BioSpin GmbH, Ettlingen, Germany). The latter consists of a 2 by 2 array of square coil elements, each sized $16 \times 16 \mathrm{~mm}^{2}$. 3D $\mathrm{T}_{1}$ mapping was performed as published previously [10] using retrospective triggering (3D IntraGate) with variable flip angles. In the current study, three different flip angles $\alpha$ were used $\left(\alpha=2^{\circ}, 8^{\circ}\right.$ and $\left.14^{\circ}\right)$. This was shown to produce highly reproducible myocardial $\mathrm{T}_{1}$ maps, in which the 95\% confidence interval for $\Delta R_{1}$ at baseline values was only $0.15 \mathrm{~s}^{-1}$. After pre-contrast $\mathrm{T}_{1}$ measurements, the same sequence was performed in black-blood mode and $\alpha=8^{\circ}$ for assessing wall thickening values. For this particular purpose, black-blood was chosen over brightblood scans, because of superior wall delineation. On the other hand, black-blood scans are less suitable for $\mathrm{T}_{1}$ mapping (see Discussion). Although the use of retrospective triggering enables reconstruction of an arbitrary number of cardiac frames, a sufficient number averages is needed for each frame. With an imaging time of 20 minutes, reconstruction of 12 cardiac frames provided good quality black-blood 3D CINE images for determining wall thickening values from end-diastolic and endsystolic frames. The total imaging time for each animal was approximately 90 minutes.

\section{Data analysis}

Pre- and post-contrast myocardial 3D $\mathrm{T}_{1}$ maps were segmented according to the AHA 17-segment model [14] using a custom-built segmentation algorithm (Mathematica 7.0, Wolfram Research, Champaign, IL, USA). Segmentation was based on the high flip angle $\mathrm{T}_{1}$-weighted scan, in which blood-myocardium contrast was highest. For each segment, mean pre- and post-contrast $R_{1}$ values were calculated $\left(R_{1}=1 / T_{1}\right)$, as well as corresponding $\Delta R_{1}$ values $\left(R_{1}{ }^{\text {post }}-R_{1}{ }^{\text {pre }}\right)$. Additionally, wall thickness (WT) of all segments was determined from the black-blood 3D IntraGate scans in both enddiastolic (ED) and end-systolic (ES) cardiac phases.
Systolic wall thickening (SWT) values were defined as $\left(\mathrm{WT}^{\mathrm{ES}} / \mathrm{WT}^{\mathrm{ED}}-1\right) * 100 \%$.

\section{Ex vivo Gd quantification}

After post-contrast CMR measurements, mice were sacrificed through cervical dislocation. From all mice in the infarct group, the heart was excised and stored in the freezer at $-80^{\circ} \mathrm{C}$ until further use. A mid ventricular slice containing the infarct area $(2.5 \mathrm{~mm}$ above the apex) was then cut from the heart using a mouse heart slicer (Zivic Instruments, Pittsburgh, PA, USA). The slice was subsequently cut into four segments (septal, posterior, lateral and anterior), each of which was weighed and put in a vial. For all segments, the Gd content (in nanograms) was determined with ICP-MS. The Gd concentration ([Gd]) was then calculated in each segment using the specific gravity of myocardial tissue $(1.05 \mathrm{~g} / \mathrm{ml})$ and the molar weight of $\mathrm{Gd}(157.25 \mathrm{~g} / \mathrm{mol})$.

\section{Statistics}

Statistical analysis was done with SPSS 17.0 (SPPS, Inc., Chicago, IL, USA) and JMP 9.0 (SAS Institute, Cary, NC, USA). All values are reported as mean \pm standard deviation. All tests were performed on the $R_{1}$ values, rather than on $T_{1}$ values. The level of statistical significance was set at $\mathrm{p}=0.05$ for all tests. Pre- and postcontrast $R_{1}$, as well as $\Delta R_{1}$ values were considered separately. For these parameters, significant differences between infarct, remote and control areas were tested with a one-way ANOVA and post-hoc Tukey test. Note that infarct segments were defined as those segments with a corresponding wall thickening value smaller than $15 \%$, whereas for remote regions, the lower threshold was set at $45 \%$. Differences in $\Delta R_{1}$ as function of wall thickening were also tested using a one-way ANOVA and post-hoc Tukey test. To correlate regional $\Delta \mathrm{R}_{1}$ values as estimated from in vivo CMR data with the Gd concentrations as measured by ex vivo ICP-MS, orthogonal regression was performed. This was preferred over linear regression, because errors in both measurement techniques should be taken into account.

\section{Results}

Figure 1 shows end-diastolic black-blood images of a representative mouse of the infarct group in four different slices reconstructed from the 3D imaging volume. The entire CINE series containing all cardiac frames is provided as a supplemental movie in Additional File 1. Blood suppression was excellent throughout the whole $3 \mathrm{D}$ volume, resulting in good delineation of the cardiac wall without presence of blood flow artifacts. Typically, akinetic regions were found in the postero- and anterolateral wall. 

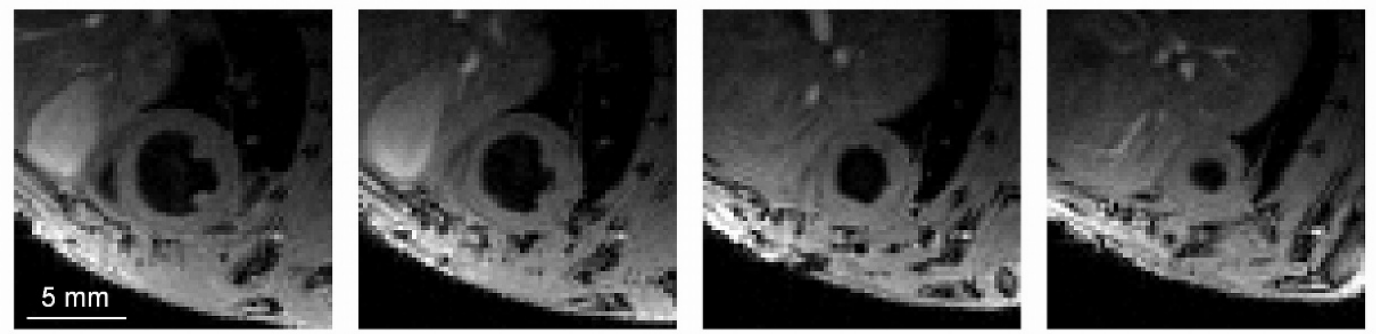

Figure 1 3D black-blood retrospectively triggered CINE images used for determination of wall thickening. 3D black-blood retrospectively triggered CINE images used for determination of wall thickening. Images from left to right represent different slices through the heart towards the apex (movie provided in Additional File 1).

Representative maps from pre- and post-contrast myocardial $\mathrm{T}_{1}$, as well as corresponding post-contrast $\mathrm{T}_{1-}^{-}$ weighted images are shown in Figure 2. Akinetic regions apparent from Figure 1 can already be distinguished in the pre-contrast $\mathrm{T}_{1}$ maps (Figure $2 \mathrm{~A}$ ) from an increase in $\mathrm{T}_{1}$. However, they are more evident in the post-contrast images from a large increase in signal intensity (Figure 2C, arrows) and corresponding significant decrease in $\mathrm{T}_{1}$ (Figure 2B).

Figure 3A shows Bull's eye plots of regional values of $\Delta \mathrm{R}_{1}\left(\mathrm{R}_{1}{ }^{\text {post }}-\mathrm{R}_{1}{ }^{\text {pre }}\right)$ and wall thickening of the same mouse as in Figure 2. The latter was divided into three categories, where SWT values $<15 \%$ and $>45 \%$ were considered 'infarcted' or 'remote', respectively $[1,15]$. Regions of low SWT matched with regions of high $\Delta \mathrm{R}_{1}$ values, induced by the presence of the paramagnetic liposomes. The mean $\Delta R_{1}$ as function of SWT is plotted in Figure $3 B . \Delta R_{1}$ decreased rapidly with increasing percentage of wall thickening, but only severely infarcted regions revealed significantly elevated $\Delta R_{1}$ values compared to remote regions. For comparison, SWT in healthy control mice was $65.0 \pm 12.3 \%$.

Statistical differences in pre-contrast $\mathrm{T}_{1}$, post-contrast $\mathrm{T}_{1}$ and $\Delta \mathrm{R}_{1}$ between infarct, remote and control regions are elaborated in Figure 4. For all parameters, there was a significant difference $(\mathrm{p}<0.01)$ between the infarct
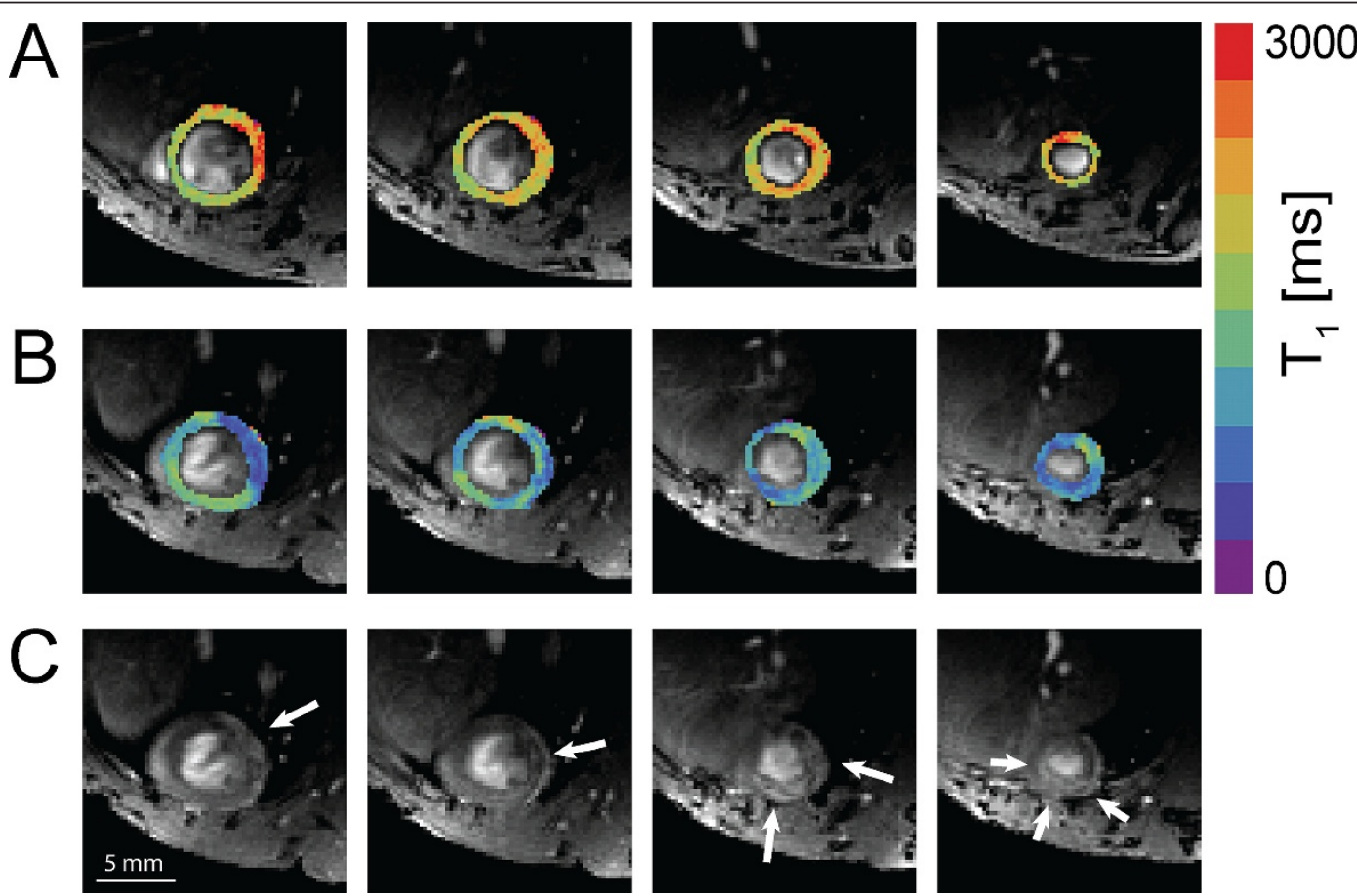

Figure 2 Pre-contrast myocardial $T_{1}(A)$, post-contrast myocardial $T_{1}(B)$, and post-contrast $T_{1}$-weighted images (C) in a mouse with myocardial infarction. Pre-contrast myocardial $T_{1}(A)$, post-contrast myocardial $T_{1}(B)$, and post-contrast $T_{1}$-weighted images $(C)$ in a mouse with myocardial infarction. Arrows mark infarct regions showing contrast enhancement in post-contrast $\mathrm{T}_{1}$-weighted imaging. Images represent the same slices as in Figure 1. 


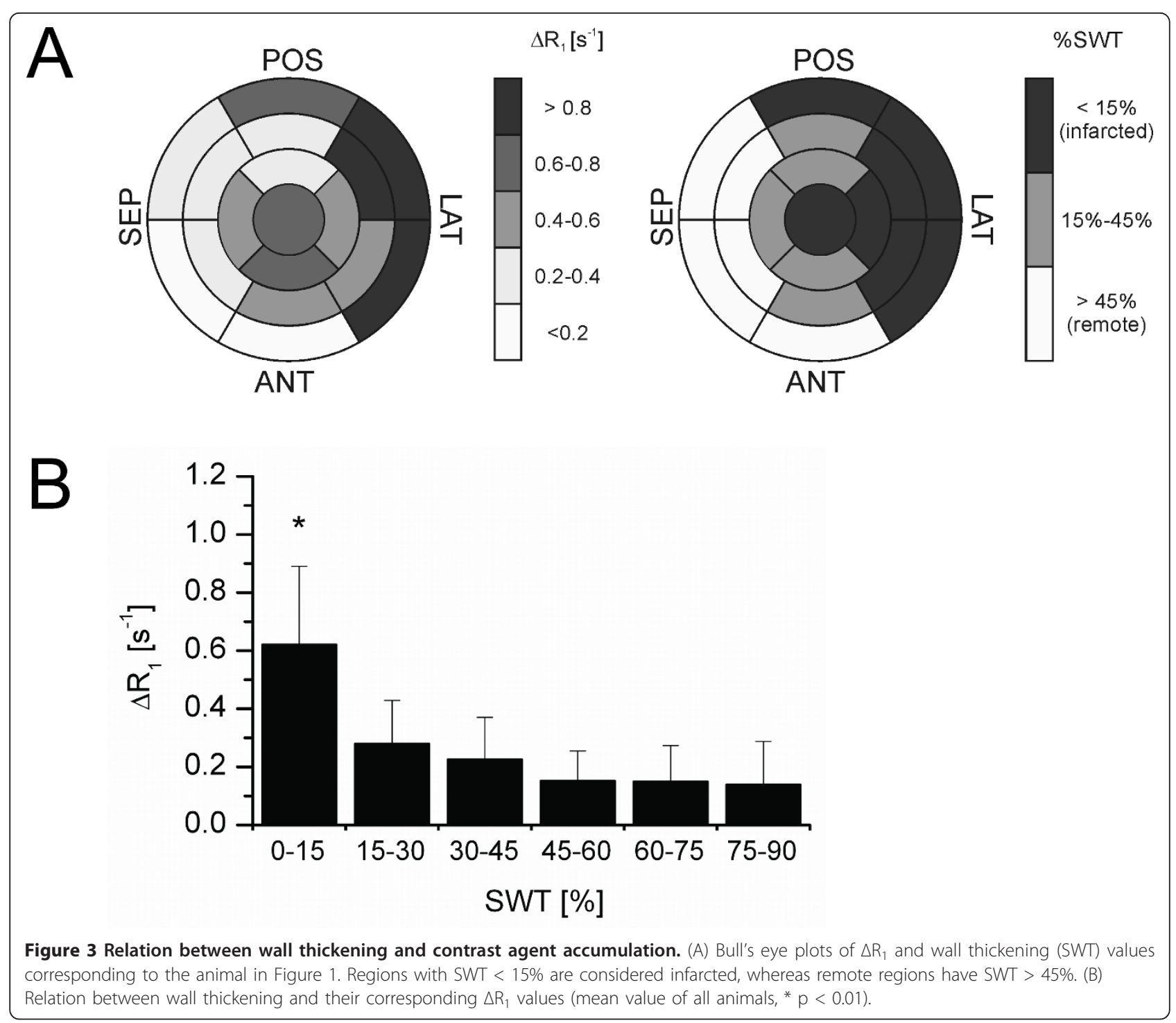

regions on the one hand and both control and remote regions on the other hand. In pre-contrast $\mathrm{T}_{1}$ scans, the mean $\mathrm{T}_{1}$ of infarcted myocardium was elevated (2030 \pm $131 \mathrm{~ms})$ as compared to remote $(1761 \pm 114 \mathrm{~ms})$ and control values $(1667 \pm 50 \mathrm{~ms})$. Post-contrast $\mathrm{T}_{1}$ values were decreased compared to pre-contrast $T_{1}$ in infarct $(910 \pm 107 \mathrm{~ms})$, remote $(1400 \pm 196 \mathrm{~ms})$ as well as control $(1203 \pm 86 \mathrm{~ms})$ regions. $\mathrm{T}_{1}$ was significantly lowest in the infarcted myocardium. The post- and pre-contrast longitudinal relaxation rate difference $\left(\Delta \mathrm{R}_{1}\right)$ was largest for the infarct region $\left(0.60 \pm 0.13 \mathrm{~s}^{-1}\right)$. However, also in remote tissue and in control animals the contrast agent resulted in a significant $\Delta \mathrm{R}_{1}\left(0.15 \pm 0.08 \mathrm{~s}^{-1}\right.$ and $0.23 \pm$ $0.05 \mathrm{~s}^{-1}$, respectively, not significantly different). Since the liposomal contrast agent does not extravasate from healthy blood vessels, these $R_{1}$ changes in remote and control tissues are likely caused by contrast agent still circulating in the blood 24 hours post injection.

Figure 5 shows the relation between $\Delta R_{1}$ values determined by in vivo CMR and Gd concentrations determined by ex vivo ICP-MS. When considering all segments that were analyzed separately (Figure 5A), the Pearson correlation coefficient $r$ was 0.6 with $R^{2}=$ 0.36 . However, a more substantial correlation was found after averaging values for each heart slice ( $\mathrm{r}=$ 0.81 ) with a corresponding $\mathrm{R}^{2}$ of 0.65 (Figure $5 \mathrm{~B}$ ). Furthermore, a small but significant offset for $\Delta R_{1}$ was found in both analyses. The slope is the effective in vivo relaxivity of the liposomal contrast agent $r_{1}=1.06$ $\mathrm{mM}^{-1} \mathrm{~s}^{-1}$. This was significantly lower than $\mathrm{r}_{1}=2.2$ $\mathrm{mM}^{-1} \mathrm{~s}^{-1}\left(\mathrm{R}^{2}=0.99\right)$ for the liposomes in suspension in vitro. 

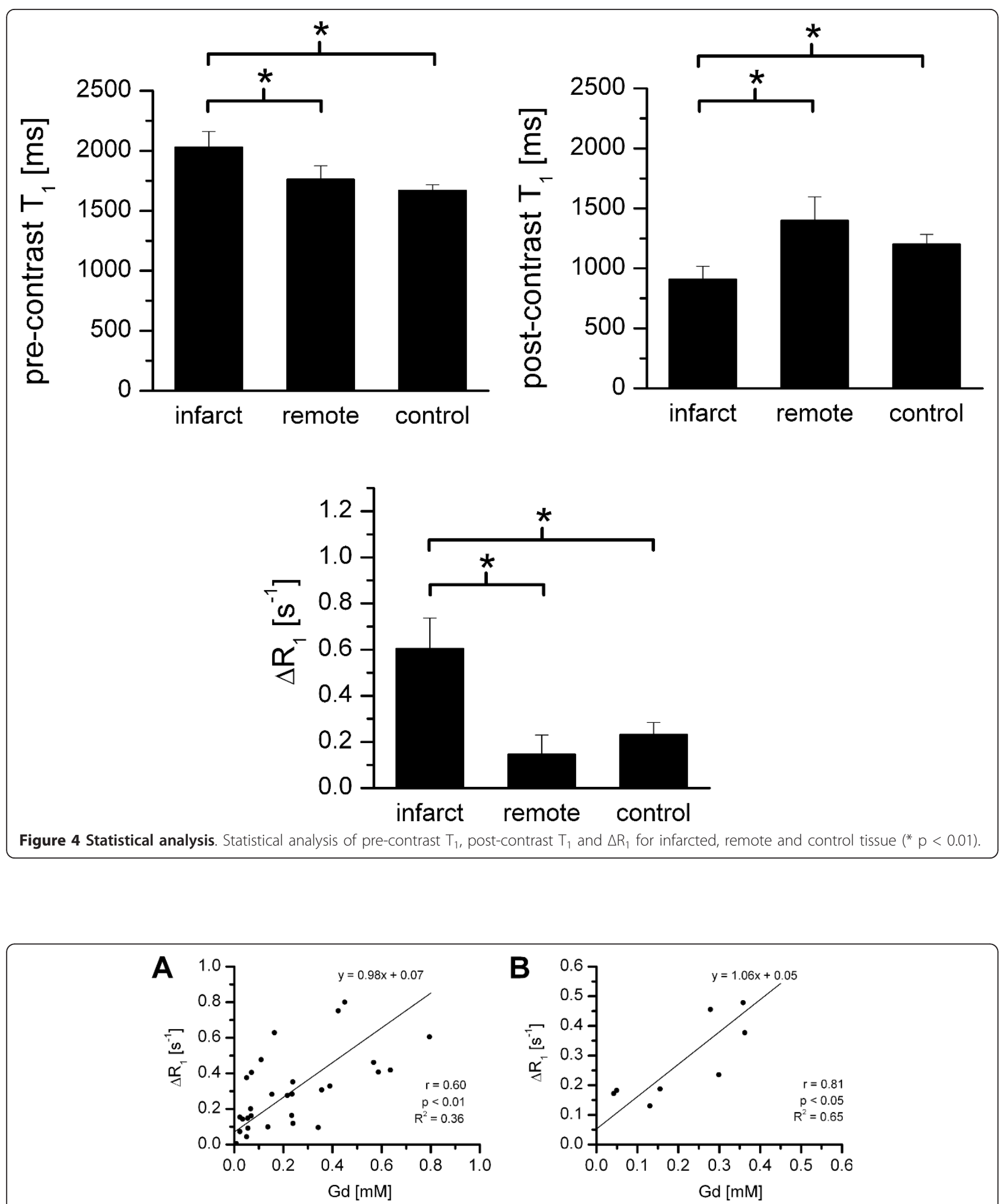

Figure $\mathbf{5}$ Correlation between regional $\Delta \mathbf{R}_{1}$ and Gd concentration. Correlation between regional $\Delta R_{1}$ values determined by in vivo $C M R$ and Gd concentration determined by ex vivo ICP-MS. The analysis was done either on all individual heart segments (A) or after averaging all values in each heart slice (B). 


\section{Discussion}

In this paper we described the use of $3 \mathrm{D} \mathrm{T}_{1}$ mapping to assess myocardial relaxation rates after ischemia/reperfusion injury before and after administration of a liposomal contrast agent. This type of contrast agent is especially known for its high relaxivity per particle and long circulation half-life [16], but also for its prolonged retention in the infarcted tissue, allowing visualization of the contrast agent many hours after administration [17-19].

In this study, we chose to perform post-contrast measurements 24 hours after contrast agent injection. The 24 hours time period allowed for sufficient accumulation of contrast agent in the infarct area, while at the same time providing sufficient clearance of the contrast agent from the blood. Similar $\Delta \mathrm{R}_{1}$ values in remote and control tissue however suggested that a small amount of contrast agent was still present in the blood. At later time points, full blood clearance would have been achieved, but probably at the expense of efflux of contrast agent from the infarction. Also, it is known that infarct development and healing is a very dynamic process, whereas pre- and post-contrast measurements are aimed at representing the same infarct status. Therefore we limited our $T_{1}$ quantification to the 24 hours time period. Previous experiments have shown a negligible mean effect of repeated measurements on $R_{1}$ [10]. In future experiments, pre- and post-contrast blood $T_{1}$ measurements could be incorporated to better quantify the influence of blood contrast agent concentration levels on myocardial $\mathrm{T}_{1}$ changes.

The black-blood protocol used in this study for functional measurements could also have been used for myocardial $\mathrm{T}_{1}$ mapping. However, although black-blood imaging was very effective in suppressing signal from the blood (see Figure 1), it does not prevent exchange of blood magnetization with the myocardium and therefore does not eliminate contamination of myocardial signal due to presence of contrast agent in the blood. Also, we have previously shown that saturation of the blood results in dramatically lower effective myocardial $\mathrm{T}_{1}$ values [10].

Based on regional $\Delta R_{1}$ values, a significantly higher uptake of contrast agent was found in infarct regions compared to remote and control regions. The identification of regions being either 'infarcted' or 'remote' was based on wall thickening values determined from functional CINE measurements (Figure 1). We chose rather conservative threshold values for wall thickening $(<15 \%$ and $>45 \%$ ) to ensure proper identification of infarct and remote areas $[1,15]$. Additionally, it was shown that the infarct area had a significantly higher $\mathrm{T}_{1}$ value already in pre-contrast scans. Previously, elevated $T_{1}$ values in infarcted myocardium have been attributed to several different mechanisms. Some studies argue that formation of myocardial edema early after ischemia results in $T_{1}$ increase due to a larger fraction of free water $[7,20]$. The increased wall thickness in the infarcted myocardium visible in end-diastolic images (Figure 1) indeed supports this theory. Others [21] claim that this increase of myocardial $T_{1}$ is more likely caused by a decrease in perfusion, based on iso-intense remote and infarcted tissue in proton density weighted images. The use of a surface coil in this study creates a signal intensity gradient in our cardiac images, which made it difficult to assess proton density and corroborate or negate the latter hypothesis.

An ongoing topic within in the field of contrastenhanced MR imaging is the actual quantification of regional contrast agent accumulation. With respect to small animal cardiac imaging, this is one of few studies attempting to correlate contrast agent induced $\Delta R_{1}$ values derived from in vivo CMR measurements with contrast agent concentrations determined by ex vivo analysis [9]. When analyzing all segments (4 in each mouse) separately, only a weak correlation was found. However, a substantial correlation was found when concentration values of all four segments within the slice were averaged. We believe that weak correlation of individual segments is caused by inaccuracies in cutting the heart slices into exactly the same segments as those of the in vivo CMR. Because of the generally sharp demarcations of the infarct, small errors in circumferential division and sectioning of the left ventricle can therefore lead to substantial differences in Gd concentrations. We therefore believe that a significant part of the variation is caused by inaccuracies of the cutting procedure. Reproducibility of regional $\mathrm{T}_{1}$ quantification of healthy myocardium using the current CMR protocol was demonstrated previously [10]. In the present study, we found the same myocardial $\mathrm{T}_{1}$ values for the control group, proving reproducibility of the method across studies. Ex vivo determined Gd concentrations correlated fairly well with in vivo $\Delta \mathrm{R}_{1}$ values. Taken together, we therefore conclude that regional quantification of contrast agent concentrations is feasible.

At this point, the mechanism of contrast agent accumulation is believed to be passive accumulation throughout the increased extracellular space by extravasation of the liposomes through leaky vasculature in the reperfused injured myocardium [22]. Regression analysis interestingly showed that the effective relaxivity of the liposomes in vivo was lower compared to that determined in vitro. Reduced relaxivity could be mediated by the cellular environment of the myocardium, partial volume effects or compartmentalization of the liposomes [23]. Compartmentalization may result from phagocytosis of liposomes by macrophages. Whether or not 
phagocytosis occurs also depends on the liposomal formulation $[18,22]$. Future studies could focus on combining regional quantification of contrast agent concentrations with more detailed histological analysis on the cellular level to reveal the exact mechanisms of uptake. Importantly, in vivo relaxivity values must be considered for correct estimation of Gd concentrations from $\Delta \mathrm{R}_{1}$ values.

\section{Conclusions}

In summary, the quantitative $\mathrm{T}_{1}$ mapping technique described in this study provides a reproducible tool to monitor the accumulation of contrast agents in contrast-enhanced studies of murine myocardial infarction. A particular field of application is that of targeted contrast agents, which are used for mapping the spatial distribution of specific biomarkers. The contrast agent relaxivity was found to be lower than that measured in vitro, which needs consideration when quantifying local contrast agent concentrations.

\section{Additional material}

Additional file 1: 3D black-blood CINE. 3D black-blood CINE movie (12 frames) for determination of regional wall thickening.

\section{List of abbreviations}

ANOVA: analysis of variance; ICP-MS: inductive coupled plasma mass spectrometry; LAD: left anterior descending; WT: wall thickness; SWT: systolic wall thickening.

\section{Acknowledgements}

The authors want to acknowledge Peter Leenders for performing surgery and Leonie Niesen for biotechnical assistance.

Funding sources

Grant sponsor: Dutch Technology Foundation STW, applied science division of NWO and the Technology Program of the Ministry of Economic Affairs; Grant number: 07952.

\section{Authors' contributions}

BC: study design, data acquisition, image analysis, statistical analysis, manuscript drafting. TG: study design, contrast agent preparation, manuscript drafting. LP: study design, manuscript drafting. KN: study design, manuscript drafting. GS: study design, manuscript drafting and principal investigator. All authors read and approved the final manuscript.

\section{Competing interests}

The authors declare that they have no competing interests.

Received: 17 May 2011 Accepted: 5 October 2011

Published: 5 October 2011

\section{References}

1. Ross AJ, Yang ZQ, Berr SS, Gilson WD, Petersen WC, Oshinski JN, French BA: Serial MRI evaluation of cardiac structure and function in mice after reperfused myocardial infarction. Magnetic Resonance in Medicine 2002, 47:1158-1168.

2. Nahrendorf M, Hiller KH, Hu K, Ertl G, Haase A, Bauer WR: Cardiac magnetic resonance imaging in small animal models of human heart failure. Medical Image Analysis 2003, 7:369-375.
3. Epstein FH: MR in mouse models of cardiac disease. NMR Biomed 2007, 20:238-255.

4. Sosnovik DE, Garanger E, Aikawa E, Nahrendorf M, Figuiredo JL, Dai GP, Reynolds F, Rosenzweig A, Weissleder R, Josephson L: Molecular MRI of cardiomyocyte apoptosis with simultaneous delayed-enhancement MRI distinguishes apoptotic and necrotic myocytes in vivo potential for midmyocardial salvage in acute ischemia. Circ - Cardiovasc Imag 2009, 2:460-467.

5. Nahrendorf M, Sosnovik D, Chen JW, Panizzi P, Figueiredo JL, Aikawa E, Libby $P$, Swirski FK, Weissleder R: Activatable magnetic resonance imaging agent reports myeloperoxidase activity in healing infarcts and noninvasively detects the antiinflammatory effects of atorvastatin on ischemia-reperfusion injury. Circulation 2008, 117:1153-1160.

6. Caravan P, Das B, Dumas S, Epstein FH, Helm PA, Jacques V, Koerner S, Kolodziej A, Shen L, Sun WC, Zhang Z: Collagen-targeted MRI contrast agent for molecular imaging of fibrosis. Angew Chem Int Edit 2007, 46:8171-8173.

7. Bohl S, Lygate C, Barnes H, Medway D, Stork L, Schulz-Menger J, Neubauer S, Schneider JE: Advanced methods for quantification of infarct size in mice using three-dimensional high-field late gadolinium enhancement MRI. Am J Physiol - Heart Circ Physiol 2009, 296:1200-1208.

8. Li W, Zhong J, Griswold MA, Yu X: Rapid T 1 Mapping of Mouse Myocardium with Saturation Recovery Look-Locker Method. In Proceedings of the 17th Annual Meeting of ISMRM, Honolulu, Hawaii, USA 2009, 4439.

9. Waghorn B, Yang YH, Baba A, Matsuda T, Schumacher A, Yanasak N, Hu TCC: Assessing manganese efflux using SEA0400 and cardiac T-1mapping manganese-enhanced MRI in a murine model. Nmr in Biomedicine 2009, 22:874-881.

10. Coolen BF, Geelen T, Paulis LEM, Nauerth A, Nicolay K, Strijkers GJ: Threedimensional $T_{1}$ mapping of the mouse heart using variable flip angle steady-state MR imaging. NMR Biomed 2010, 24:154-162.

11. Mulder WJM, Strijkers GJ, Griffioen AW, van Bloois L, Molema G, Storm G, Koning GA, Nicolay K: A liposomal system for contrast-enhanced magnetic resonance imaging of molecular targets. Bioconjugate Chem 2004, 15:799-806.

12. Rouser G, Fkeischer C, Yamamoto A: Two dimensional thin layer chromatographic seperation of polar lipids and determination of phospholipids by phosphorus analysis of spots. Lipids 1970, 5:494-496.

13. Deichmann $R$, Haase $A$ : Quantification of $T_{1}$ values by snapshot-FLASH NMR imaging. J Magn Reson 1992, 96:608-612.

14. Hundley WG, Bluemke D, Bogaert JG, Friedrich MG, Higgins CB, Lawson MA McConnell MV, Raman SV, van Rossum AC, Flamm S, et al: Society for Cardiovascular Magnetic Resonance guidelines for reporting cardiovascular magnetic resonance examinations. J Cardiovasc Magn $R$ 2009, 11:5.

15. Ichikawa Y, Sakuma H, Suzawa N, Kitagawa K, Makino K, Hirano T, Takeda K: Late gadolinium-enhanced magnetic resonance imaging in acute and chronic myocardial infarction - Improved prediction of regional myocardial contraction in the chronic state bv measurinz thickness of nonenhanced myocardium. Journal of the American College of Cardiology 2005, 45:901-909.

16. van Bochove GS, Paulis LEM, Segers D, Mulder WJM, Krams R, Nicolay K, Strijkers GJ: Contrast enhancement by differently sized paramagnetic MRI contrast agents in mice with two phenotypes of atherosclerotic plaque. Contrast Media Mol Imaging 6:35-45

17. Geelen T, Paulis LEM, Nicolay K, Strijkers GJ: Passive targeting of cardiac ischemia/reperfusion injury with high molecular weight paramagnetic contrast agents. Magn Reson Mater Phy 2009, 22(Supplement 1).

18. Geelen T, Yeo SY, Paulis LEM, Coolen BF, Nicolay K, Strijkers GJ: In vivo MR imaging of macrophages in cardiac ischemia/reperfusion injury with paramagnetic phosphatidylserine-containing liposomes. Proceedings of the 19th Annual Meeting ISMRM; Montreal, Canada 2011, 323.

19. Paulis LEM, Geelen T, Kuhlmann M, Coolen BF, Nicolay K, Strijkers GJ: Contrast-enhanced cardiac MRI of vascular remodeling after myocardial infarction using lipid-based nanoparticles. Proceedings of the 19th Annual Meeting ISMRM; Montreal, Canada 2011, 1358.

20. Messroghli D, Walters K, Plein S, Sparrow P, Friedrich MG, Ridgway JP, Sivananthan MU: Myocardial $T_{1}$ mapping: application to patients with acute and chronic myocardial infarction. Magn Reson Med 2007, 58:34-40. 
21. Vandsburger MH, Janiczek RL, Xu YQ, French BA, Meyer CH, Kramer CM, Epstein FH: Improved arterial spin labeling after myocardial infarction in mice using cardiac and respiratory gated look-locker imaging with fuzzy c-means clustering. Magn Reson Med 2010, 63:648-657.

22. Geelen T, Paulis LEM, Coolen BF, Nicolay K, Strijkers GJ: Passive targeting of paramagnetic lipid-based contrast agents to acute mouse cardiac ischemia/reperfusion injury. Proceedings of the 19th Annual Meeting ISMRM: Montreal, Canada 2011, 1356.

23. Strijkers GJ, Hak S, Kok MB, Springer CS, Nicolay K: Three-compartment T, relaxation model for intracellular paramagnetic contrast agents. Magn Reson Med 2009, 61:1049-1058.

doi:10.1186/1532-429X-13-56

Cite this article as: Coolen et al.: Regional contrast agent quantification

in a mouse model of myocardial infarction using $3 \mathrm{D}$ cardiac $\mathrm{T}_{1}$

mapping. Journal of Cardiovascular Magnetic Resonance 2011 13:56.

\section{Submit your next manuscript to BioMed Central} and take full advantage of:

- Convenient online submission

- Thorough peer review

- No space constraints or color figure charges

- Immediate publication on acceptance

- Inclusion in PubMed, CAS, Scopus and Google Scholar

- Research which is freely available for redistribution

Submit your manuscript at www.biomedcentral.com/submit 